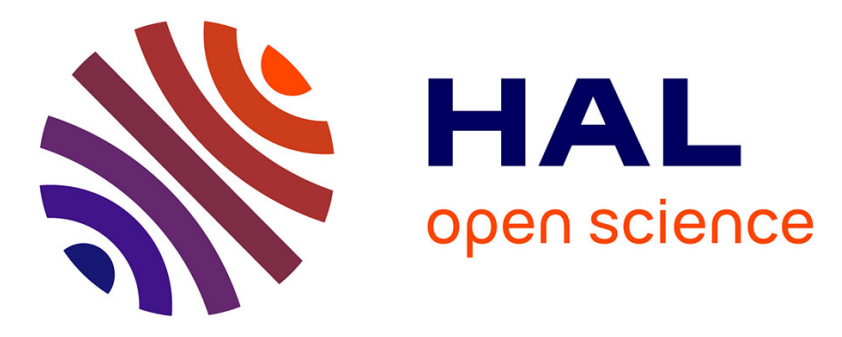

\title{
Hydrodynamic effects induced by nanosecond repetitive pulsed discharges
}

\author{
Sergey Stepanyan, Nicolas Minesi, Erwan Pannier, Gabi-Daniel Stancu, \\ Christophe O Laux
}

\section{> To cite this version:}

Sergey Stepanyan, Nicolas Minesi, Erwan Pannier, Gabi-Daniel Stancu, Christophe O Laux. Hydrodynamic effects induced by nanosecond repetitive pulsed discharges. 2018 AIAA Aerospace Sciences Meeting, Jan 2018, Kissimmee, United States. 10.2514/6.2018-0930 . hal-01866398

\section{HAL Id: hal-01866398 \\ https://hal.science/hal-01866398}

Submitted on 24 Feb 2020

HAL is a multi-disciplinary open access archive for the deposit and dissemination of scientific research documents, whether they are published or not. The documents may come from teaching and research institutions in France or abroad, or from public or private research centers.
L'archive ouverte pluridisciplinaire HAL, est destinée au dépôt et à la diffusion de documents scientifiques de niveau recherche, publiés ou non, émanant des établissements d'enseignement et de recherche français ou étrangers, des laboratoires publics ou privés. 


\title{
Hydrodynamic effects induced by nanosecond repetitive pulsed discharges
}

\author{
Sergey Stepanyan, Nicolas Minesi, Erwan Pannier, Gabi D. Stancu and Christophe O. Laux \\ EM2C Laboratory, CNRS UPR288, CentraleSupélec, Université Paris Saclay, 91190 Gif-sur-Yvette, France
}

The intense energy released in a burst of nanosecond sparks is shown to induce hydrodynamic effects over large spatial scales of the order of a centimeter. This gas motion transports the active species produced by the discharge into the surrounding space. The present work is dedicated to the detailed measurements of the energy deposited in plasma and its redistribution in space through hydrodynamic degrees of freedom.

\section{Introduction}

$\mathrm{N}$ anosecond Repetitively Pulsed (NRP) discharges have been developed for various applications such as PlasmaAssisted Ignition (PAI) [1-5], lean flame stabilization [6-8], aerodynamic flow control [9-12], biodecontamination and nanomaterial synthesis [13-15].

The peculiarity making nanosecond plasmas attractive for these applications is the fast gas heating phenomenon and its consequences [16]. Fast heating occurs in air-containing mixtures due to dissociative quenching reactions between molecular oxygen and electronically excited nitrogen molecules produced by electron impact during the discharge [17,18]. The ultrafast gas heating in atmospheric pressure air plasmas occurs within a few tens of nanoseconds in the discharge afterglow. Nearly full dissociation of molecular oxygen [18-24] is another feature of nanosecond plasmas which provides large amounts of reactive chemical species.

A known consequence of the fast gas heating by nanosecond plasmas is the formation of shock waves and hydrodynamic effects $[25,26]$. It has been shown in [25] that the thin channel of gas excited by a single spark propagates over a few millimeters within tens of microseconds. It has been also demonstrated for combustible mixtures of propane [27-32] that the burst of nanosecond sparks can cause wrinkling of the flame front and lead to its acceleration due to the hydrodynamic motion induced by nanosecond plasma. The effect of nanosecond sparks on the formation of ignition kernel was particularly considered in [33,34].

The modeling performed by Castela et al [35] showed that the hot gas core produced by the spark develops into an expanding torus of reactive hot gas, and that the fresh gas recirculates inside the interelectrode region. This was confirmed by LIF and by PIV (particle image velocimetry) in [36].

Hydrodynamic expansion following the nanosecond sparkshould be studied because the energy deposited in the plasma redistributes in space during a period typically shorter than the ignition delay time. This means that preformation of the ignition kernel occurs due to hydrodynamic motion induced by the intense heat release in the nanosecond plasma. Parametric studies are required to understand these phenomena, i.e expansion dynamics should be studied for different gas mixtures, speeds of external flow, distances between the electrodes and different frequencies of applied pulses in the case of repetitive discharges.

The present work is focused of the investigation of hydrodynamic effects induced by nanosecond repetitive sparks. The main part of experimental information was obtained by electrical measurements and optical diagnostics including Planar Laser-Induced Fluorescence (PLIF), Schlieren, emission spectroscopy and fast imaging.

\section{Experimental setup}

The majority of experiments presented here were performed for nanosecond sparks initiated by overvoltage pulses between pin-to-pin electrodes. The pressure was always $1 \mathrm{~atm}$. The pins used in the present work had tips of conic shape with a radius of curvature of about $50 \mu \mathrm{m}$ and were made of stainless steel. It should be noted that for different pin-electrodes (i.e. for various pin-tip radii of curvature) no difference was observed, neither in the energy deposition nor in the hydrodynamic effects induced by the plasma [37]. So the results presented here for pin-to-pin geometry can be considered to be quite general. Some additional experiments were performed in pin-to-plane geometry. The distance between the electrodes was in the range 0.5-7 mm. The spark discharges were created with an FID FPG 30-100MC4K generator producing pulses of 10-ns duration, amplitudes up to $30 \mathrm{kV}$ and Pulse 
Repetition Frequency (PRF) up to $100 \mathrm{kHz}$. The electrical characteristics of the discharge were monitored by high voltage probes (Lecroy PPE), current probes (Pearson model 6585) connected to the electrodes system, and a 1-GHz digitizing oscilloscope (LeCroy Wavepro 7100). The procedure for energy measurements in this configuration can be found elsewhere [13].

Comparison of the nanosecond discharge was performed with an AUDI coil system widely used as a car igniter. For this system, discharge breakdown occurs during the first tens of nanoseconds. After this stage the spark discharge transforms into an arc that lasts up to a few milliseconds. The typical value of total energy deposition in this case is a few tens of $\mathrm{mJ}$.

Figure 1 presents a schematic of the experimental setup used to monitor the long discharge afterglow. Schlieren diagnostics were combined with PLIF, emission spectroscopy, and emission imaging. This set of optical diagnostics allows characterizing the distribution of the gas number density and the production of active species.

The hydrodynamic effects induced by ns sparks were monitored with Schlieren diagnostics. A Cavilux light source was used to provide short light pulses ( 10 ns) at high frequencies (up to $900 \mathrm{kHz}$ ). The light produced by the source was collimated with a 1-m focal-length plano-convex lens directed to the discharge location. Then the light partially deflected due to gradients of gas number density was directed to a spherical mirror of 2-m focal length. A knife-edge was placed at the focal spot of the mirror to cut $80 \%$ of the light produced by the source. A second spherical mirror of 2-m focal length was placed between the knife-edge and the CCD camera in order to provide a 1.8 magnification. An intensified fast camera (Photron SA-X2) was used to record either Schlieren images or spontaneous emission of the active species produced in the plasma.

The spatial distribution of active species produced in the discharge, such as $\mathrm{OH}$ and $\mathrm{NO}$, was characterized by monitoring the PLIF intensity from the area corresponding to the discharge afterglow at temporal scales of $200 \mu \mathrm{s}$. A frequency-doubled dye laser (Continuum ND6000) with Rhodamine 590 dye, pumped by a Nd:YAG laser (Continuum) at $532 \mathrm{~nm}$, was used to excite the Q1(6) transition of $\mathrm{OH}\left[\left(X, v^{\prime \prime}=1\right) \rightarrow\left(A, v^{\prime \prime}=1\right)\right]$ at $282.935 \mathrm{~nm}$. LIF emission was collected through band pass filters (CORION 10BPF10-310 centered at $310 \mathrm{~nm}$ with $11 \mathrm{~nm}$ FWHM for $\mathrm{OH}^{*}$ ) placed in front of the CCD camera. The laser sheath for PLIF was formed by a combination of cylindrical $(\mathrm{f}=15 \mathrm{~cm})$ and spherical $(\mathrm{f}=10 \mathrm{~cm})$ lenses. The height of the sheath was $10 \mathrm{~mm}$ and its thickness was $0.2 \mathrm{~mm}$. The laser frequency was $10 \mathrm{~Hz}$, with a pulse width of 6-ns FWHM.

The discharge, laser and cameras were synchronized with a gate-and-delay generator (BNC Model 575).

\section{Experimental results}

Hydrodynamic effects induced by sparks are governed by the dynamics of energy release in the plasma. Therefore, the monitoring of hydrodynamic effects by optical diagnostics must be coupled with the measurements of the energy deposited in plasma. Figures 2a-f present the results of energy measurements obtained for a burst of eight pulses for various conditions in ambient air. For all the presented results the discharges were initiated by pulses of $10 \mathrm{~ns}$ duration and of voltage amplitude $30 \mathrm{kV}$ in open circuit. Burst frequencies we re within the $5-100 \mathrm{kHz}$ range. The speed of the external air flow was varied between 0 and $40 \mathrm{~m} / \mathrm{s}$.

One can see that for higher burst frequencies $(>5 \mathrm{kHz})$ the energy deposited in the second pulse of the burst is always lower than in the first one. This is attributed to the change of gas density and gas content before the second pulse by the discharge initiated in the first pulse. At lower burst frequencies $(\sim 5 \mathrm{kHz})$ the energy deposited in the second and other pulses is practically the same as in the first pulse. Therefore, at the considered conditions the effect of discharge coupling occurs at frequencies higher than $5 \mathrm{kHz}$. In case of coupling the energy deposited in each new spark stabilizes after a few first pulses in the burst.

The same dependence of the energy deposition on the burst frequency is observed for pure argon and oxygen (see figure $2 \mathrm{~g}$. As the result the discharges initiated in these gases induce hydrodynamic affects at similar temporal and spatial scales, as demonstrated in [37].

A summary of the results on energy measurements is presented in figure $2 \mathrm{~h}$. The average energy deposited per pulse in the burst changes quite smoothly for inter-electrode gap distances of 2-7 mm, for the flow speed range 0-40 $\mathrm{m} / \mathrm{s}$, and for all gases considered (air, oxygen, nitrogen, argon). Values measured for all the considered conditions are within the range 1.5-3.5 mJ per pulse in the burst. It should be noted that for all the considered conditions a transition streamer arc described in [38] occurs within a nanosecond or so. Figure 3 demonstrates images of total emission before and after streamer-arc transition for a single nanosecond spark initiated in ambient air by a $10 \mathrm{~ns}$ pulse of $30 \mathrm{kV}$. Discharge emission before the transition is mostly due to molecular nitrogen and after the transition is due to ions of atomic nitrogen. Radial profiles of the discharge before and after the transition to the arc are presented in figure 4. One of the main peculiarities of the transition to the arc is the temperature rise up to $40000 \mathrm{~K}$ 
at nanosecond scale (see [38] for more details). This provides high level temperature gradients and as a consequence shock waves and hydrodynamic motion of the gas in the area around the electrodes.

The results of the energy measurements presented in figure 2 for the case with external flow of air may look confusing because the energy decreases after the first pulse and then increases after a few pulses. It has been already mentioned that the energy deposition depends on the gas density and gas content. In the case of the external gas flow it also depends on the flow speed. The gas treated by a discharge is advected by the flow. Depending on the discharge frequency and the flow speed, the next discharge will be either initiated as a relatively straight channel between the pins or as a curved channel passing through the active medium created in the previous pulse and advected by the flow before the new one. Figure 5 presents the images of total emission for two cases: the discharge in ambient quiescent air and in an external air flow of $40 \mathrm{~m} / \mathrm{s}$. In both cases the discharge was initiated by a burst of $30 \mathrm{kV}$ pulses (10 ns duration each) at $100 \mathrm{kHz}$. The external air flow was directed perpendicularly to the pin electrode axis. One can see that the nanosecond spark placed in the external gas flow passes in each new pulse through the previously created active medium. This continues until the spark channel length becomes too long and the spark extinguishes and restarts as a straight channel again. At the considered conditions, the extremity of the curved spark channel can be at $1.5 \mathrm{~cm}$ from the electrode axis before the discharge restarts again between the electrodes. The visible volume of the discharge is also significantly larger than in the first pulse due to both length and thickness of the spark channel. All considered factors affect the energy deposition dynamics and cause the results presented in figure 2 .

The high reproducibility of single-pulse nanosecond discharges allows to acquire phase-locked images for PLIF and Schlieren diagnostics. It has also been verified that the hydrodynamic effects induced by nanosecond sparks at given conditions are also reproducible up to times of the order of milliseconds. This allowed scanning with PLIF the shape of the active medium arising in the discharge afterglow of a single nanosecond spark.

Hydrodynamic motion induced by a single nanosecond spark has already been described before [25,27,33]. Figure 6 demonstrates typical Schlieren (top row) and OH PLIF (bottom row) images obtained in the afterglow of a single spark initiated in air by a single pulse of 10-ns duration and 30-kV amplitude. The gap between the pin-to-pin electrodes in this case was $1 \mathrm{~mm}$. Each Schlieren image consists of two parts that correspond to different amounts of light cut by the knife-edge. Approximately 30 percent of light produced by a Schlieren light source was cut for the left-side pictures and 80 percent for the right-side pictures. Therefore, for the considered conditions the left-side pictures present well the gradients corresponding to the hot gas core expanding after the spark, and the right-side pictures are representative of the density gradients corresponding to the shock wave induced by the plasma. One can see from figure 6 how the intense energy release in a thin plasma channel induces a shock wave and the following expansion of the active medium. At approximately three microseconds the shock wave degrades to a sound wave and detaches from the active medium. At this moment the expansion speed of the active medium becomes about 50 $\mathrm{m} / \mathrm{s}$ per second and decreases to $20 \mathrm{~m} / \mathrm{s}$ in the next $100 \mu \mathrm{s}$. Figure 7 demonstrates the Schlieren results obtained for the same conditions as for figure 6 , but for a longer temporal scale. The result is practically identical for various conditions [25,27,33], and is valid for a single submicrosecond spark initiated between pin-to-pin electrodes at normal conditions: about $100 \mu \mathrm{s}$ after the discharge, the scale of the expanded active medium is about $5 \mathrm{~mm}$. Then the expansion becomes diffusive $(<1 \mathrm{~m} / \mathrm{s})$ and the active medium practically does not change in shape before its complete decay.

The Schlieren images presented in figure 7 show that in the case of a burst of two pulses the result will be different for the period between pulses of $20 \mu \mathrm{s}(50 \mathrm{kHz})$ and $100 \mu \mathrm{s}(10 \mathrm{kHz})$. Schlieren images presented in figure 7 show that the gas density near the electrode tips at $20 \mu$ s after the spark is lower than at $100 \mu$ s. The gas content at these moments is obviously also different. The last two statement correlate well with energy measurements presented above (see figure 2a). For multiple discharges (NRP discharge), the shape of the expanded hot gas corresponds to a superposition of single discharges. It strongly increases with the number of pulses in the burst. Figures 8 present Schlieren images obtained for a burst of 30 sparks in ambient air initiated at $30 \mathrm{kHz}$. The duration of each pulse was $10 \mathrm{~ns}$ and the voltage amplitude was $30 \mathrm{kV}$. The interelectrode distance was $2 \mathrm{~mm}$ for the image on the left and $7 \mathrm{~mm}$ for the image on the right. The total energy deposited in the burst was $60 \mathrm{~mJ}$ for the 2-mm gap and $90 \mathrm{~mJ}$ for the 7-mm gap. The estimated volume was $0.3 \mathrm{~cm}^{3}$ for the 2-mm gap and $0.9 \mathrm{~cm}^{3}$ for the 7-mm gap. Therefore, the average density was $0.2 \mathrm{~mJ} / \mathrm{mm}^{3}$ for the $2-\mathrm{mm}$ gap and $0.1 \mathrm{~mJ} / \mathrm{mm}^{3}$ for the $7-\mathrm{mm}$ gap. It should be noted that both cases correspond to volumes that are significantly larger that the ignition kernel produced by a conventional spark ignition system (for more details see [37]), whereas the volumetric energy density is still sufficient for ignition of combustible mixtures. Thus NRP can be considered as an efficient tool to redistribute spatially the energy deposited in the plasma during a period shorter than a typical ignition delay time. 


\section{Conclusions}

We have shown how the energy deposited in a repetitive nanosecond spark depends on different factors such as gas content, pulse frequency, interelectrode distance and speed of external flow. For all the cons idered conditions the values of the energy deposited per pulse were within the range 1.5-3.5 mJ. It has also been demonstrated that varying parameters of nanosecond repetitive spark one can tailor the spatial redistribution of the energy deposited in the plasma and the volume occupied by the active medium. Thus repetitive nanosecond sparks are a useful tool for the ignition of lean mixtures and the stabilization of lean flames.

\section{Acknowledgments}

This work was supported by the FAMAC project (Grant no. ANR-12-VPTT-0002) and the PLASMAFLAME project (Grant no.ANR-11-BS09-0025). The authors thank Dr. Denis Packan (ONERA) for lending the light source for Schlieren measurements.

\section{References}

[1] Pancheshnyi S et al 2006 IEEE Transactions on Plasma Science 34 2478-2487

[2] Singleton D et al 2011 J. Phys. D: Appl. Phys. 44022001

[3] Starikovskiy A et al 2013 Prog. Energy Combust. Sci. 39 61-110

[4] Starikovskaia S 2014 J. Phys. D: Appl.Phys. 47353001

[5] Ju Y et al 2015 Progress in Energy and Combustion Science 48 21-83

[6] Barbosa S et al 2015 Phil. Trans. R. Soc. A 37320140335

[7] Pilla G et al 2006 IEEE Transactions on Plasma Science 34 2471- 2477

[8] Hyungrok D et al 2008 IEEE Transactions on Plasma Science 36(6) 2898-2904

[9] Miles R et al 2009 Eur. Phys. J. Appl. Phys. 4722802

[10] Benard N et al 2008 AIAA Journal 46 (9) 2293-2305

[11] Adamovich I et al 2009 Plasma Sources Sci. Technol. 18034018

[12] Opaits D 2010 Ph.D. Dissertation, Princeton University

「131 Pai D et al 2013 Scientific Reports 31221

「14] Chen Z et al. 2011 Nano Lett. 11 4168-4175

[15] Ostrikov K 2011 J. Phys. D-Appl. Phys. 44174003

[16] Pai D et al 2010 Plasma Sources Science and Technology 19065015

[17] Stancu Get al 2010 J. Phys. D: Appl. Phys. 43124002

[18] Rusterholtz D et al 2013 J. Phys.D: Appl. Phys 46464010

[19] Popov N 2001 Plasma Physics Reports 27(10) 886-896

[20] Flitti A et al 2009 Eur. Phys. J. Appl. Phys. 4521001

「21] Aleksandrov 2010 J. Phys. D: Appl. Phys 43255201

[22] Stancu G et al 2010 J. Phys. D: Appl. Phys.43 124002

[23] Lo A et al 2012 Appl Phys B Lasers O 107(1) 229-242

[24] Klochko et al 2015 Plasma Sources Science and Technology 24(2) 025010

[25] Kono M et al 1989 Symp. (Int.) Combust. 221643

[26] Leonov S et al 2007 Physics of Plasmas 14123504

[27] Xu D et al 2011 Appl. Phys. Lett. 99121502

[28] Xu D et al 2014 J. Phys. D: Appl. Phys. 47235202

[29] Lo A et al 2014 Journal of Physics D: Applied Physics 47115201

[30] Lo A et al 2014 Journal of Physics D: Applied Physics 47115202

[31] Xu D $2013 \mathrm{PhD}$ thesis December, Ecole Centrale, Paris

[32] Xu D et al 2016 Plasma Chemistry and Plasma Processing 36 (1) 309-327

[33] Bane S et al 2015 Combustion and Flame 162(2) 462-469

[34] Xu D et al 2016 Plasma Chem Plasma Process 36 309-327

[35] Castela $\mathrm{M}$ et al 2016 Proceedings of the Combustion Institute 000 1-9

[36] Seydou A et al 2016 18th International Symposium on the Application of Laser and Imaging Techniques to Fluid Mechanics, Lisbon, Portugal, July 4-7

[37] Stepany an S et al 2017 Plasma Sources Science and Technology 26 04LT01

[38] Lo A et al 2017 Plasma Sources Science and Technology 26045012 


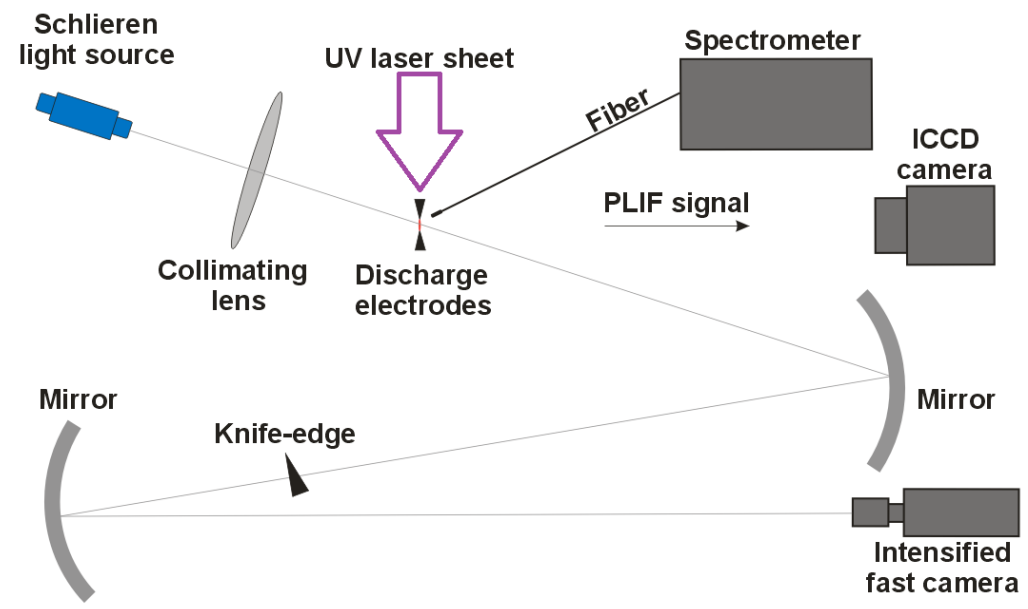

Figure 1. Experimental setup. Combination of synchronized PLIF and Schlieren diagnostics. The focal length of the lenses for Schlieren is $1 \mathbf{m}$. Both spherical mirrors have focal distances of $\mathbf{2} \mathbf{~ m}$.
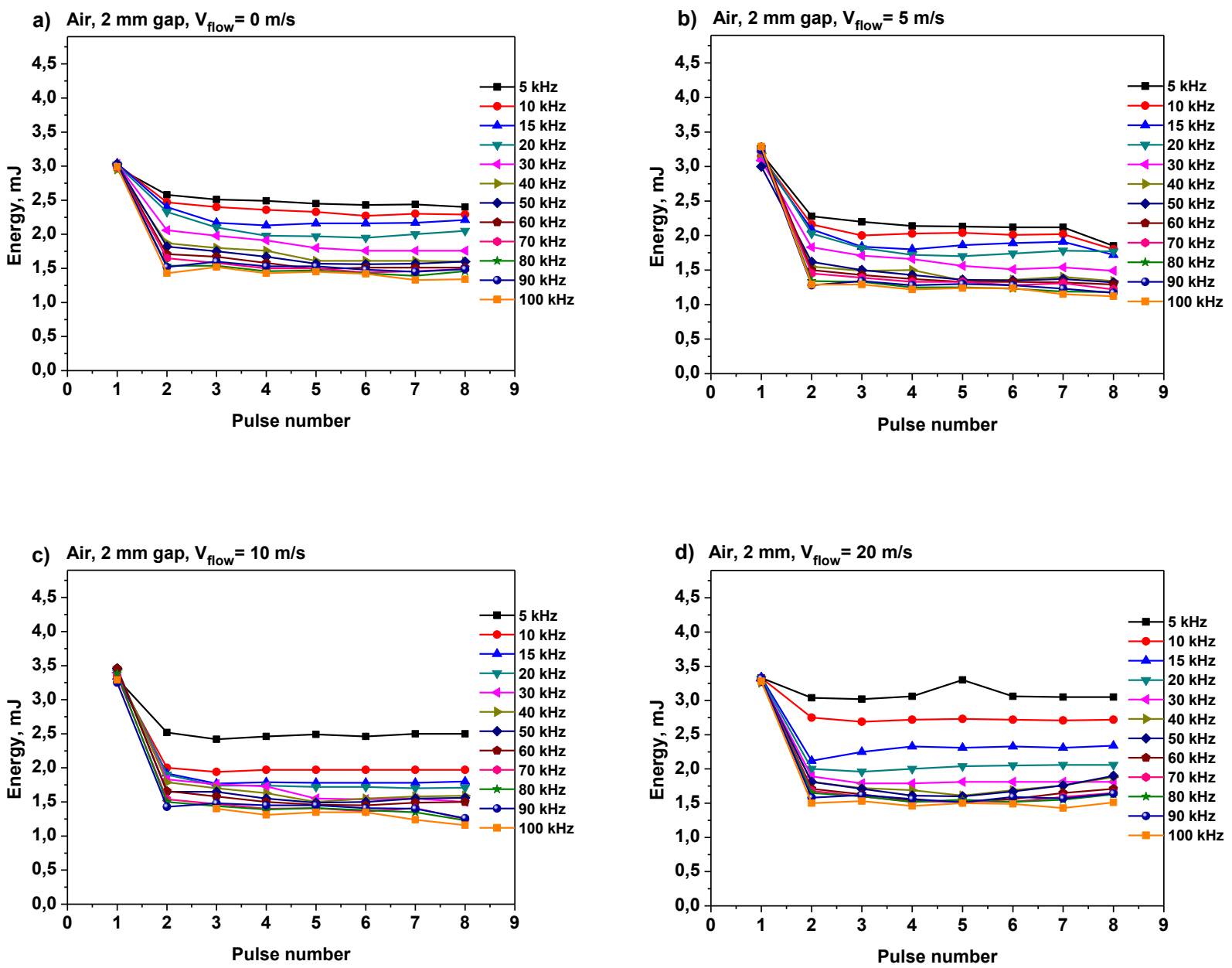

5

American Institute of Aeronautics and Astronautics 

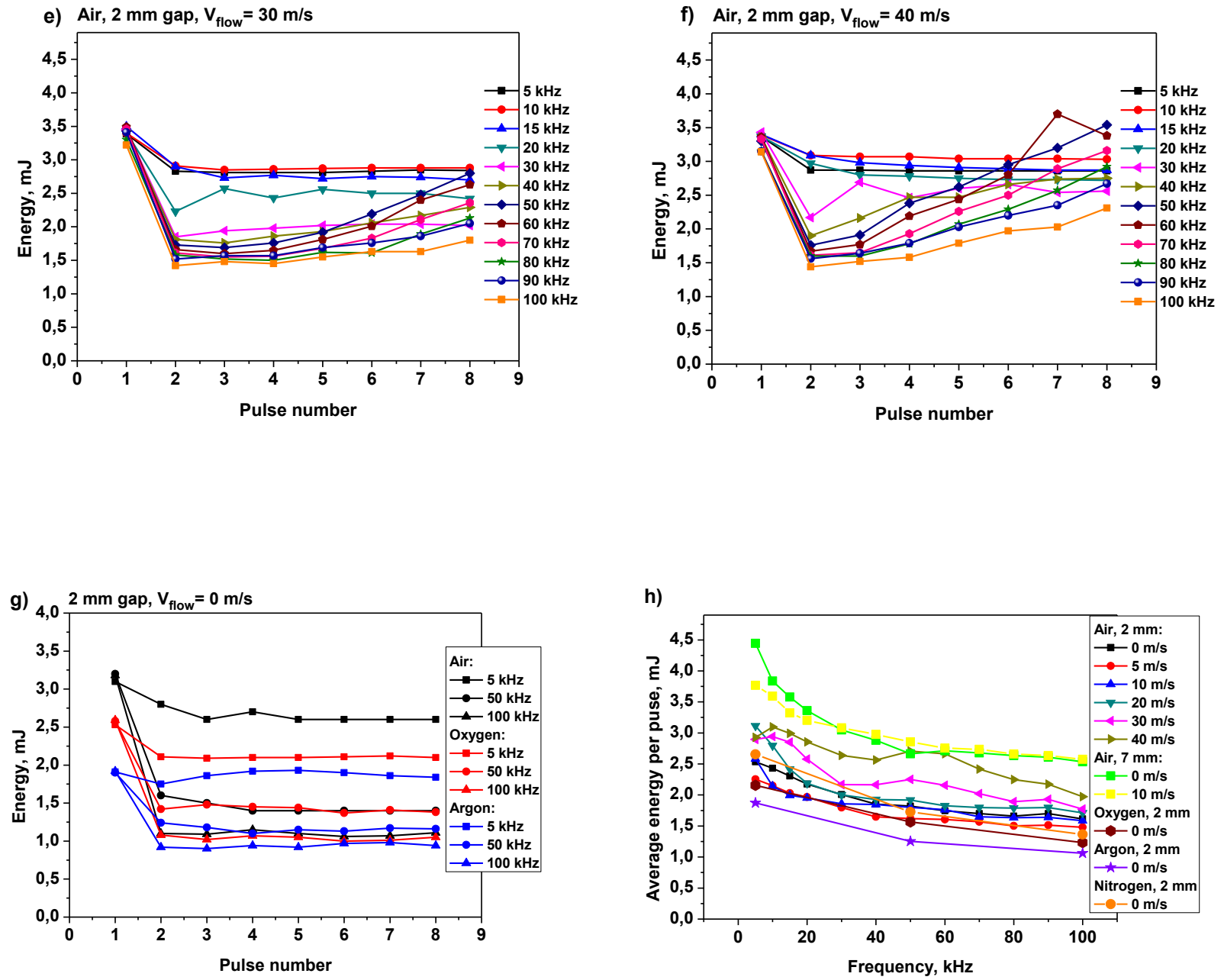

Figure 2. Energy deposited in a burst of sparks initiated in ambient air by 8 pulses $(10 \mathrm{~ns}, 30 \mathrm{kV})$ at different frequencies. The distance between the electrodes was $2 \mathrm{~mm}$.

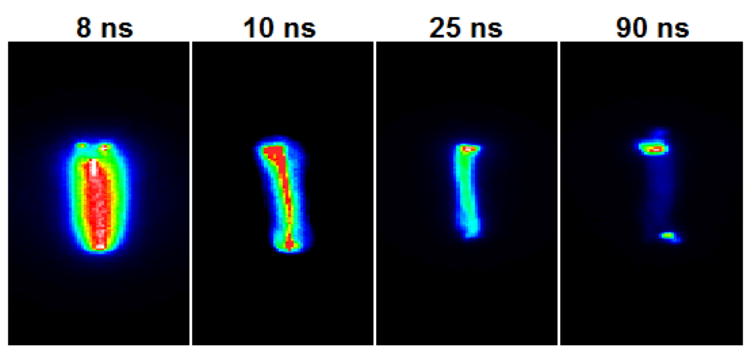

Figure 3. Total emission produced by a single nanosecond sparkinitiated in ambient air by a $10 \mathrm{~ns}$ pulse of $30 \mathrm{kV}$. The distance between the electrodes was $1 \mathrm{~mm}$. Transition streamer-arc occurs at 8-10 ns. Camera gate was $2 \mathrm{~ns}$. 


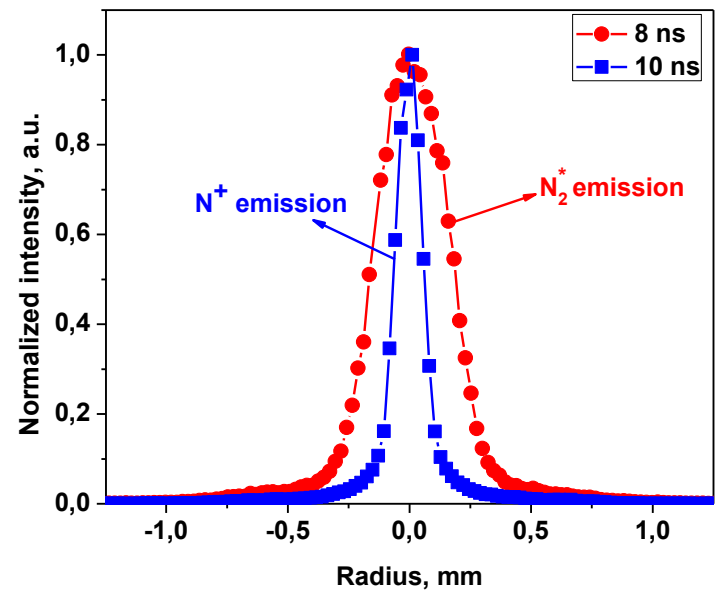

Figure 4. Profiles obtained by total emission produced by a single nanosecond sparkbefore (red eolor filled circles) and after (blue filled barssquares) transition to the arc. Discharge conditions are the same that as for figure 3 (ambient air, $10 \mathrm{~ns}$ pulse, $30 \mathrm{kV}$ ).

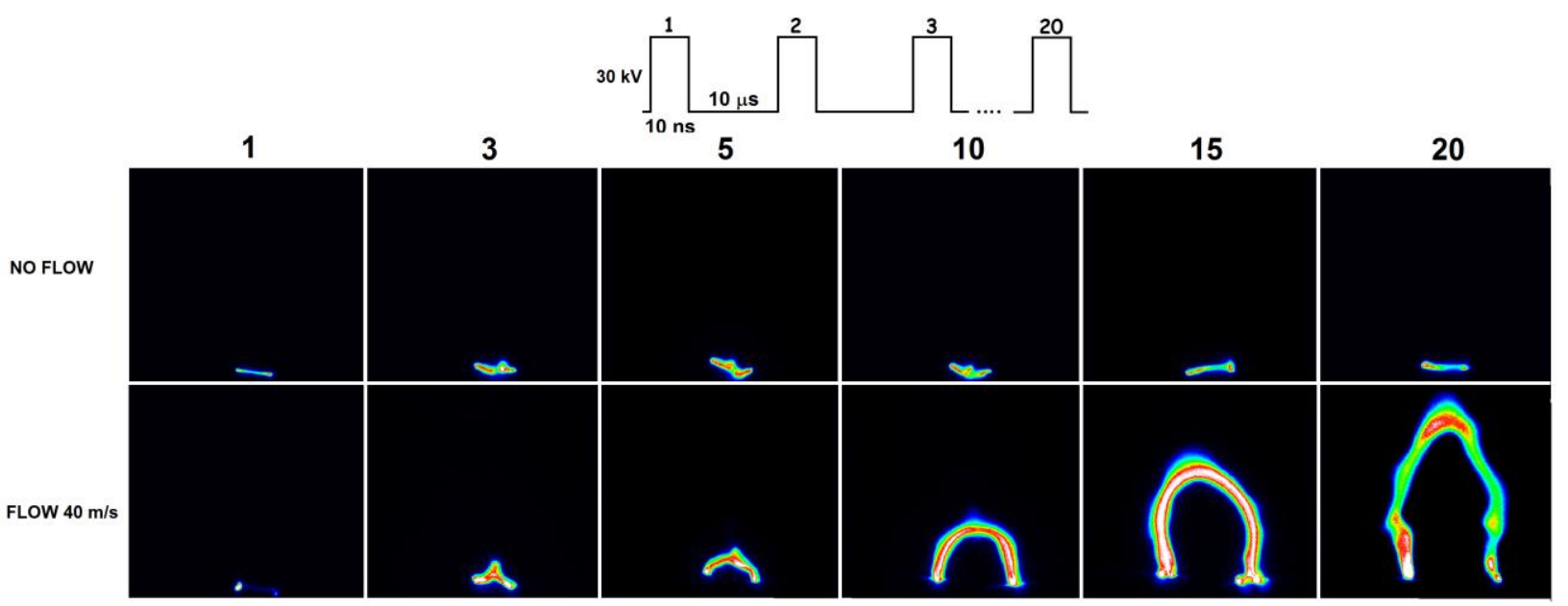

Figure 5. Comparison of total emission produced by a burst of nanosecond sparks. The Ppulse number is marked en at the top. The discharge was initiated in ambient air b $\mathrm{by}$ a burst of 20 pulses of 10--ns duration each and of 30--kV amplitude. Burst frequency was $100 \mathrm{kHz}$. The distance between the electrodes was $2 \mathrm{~mm}$. 


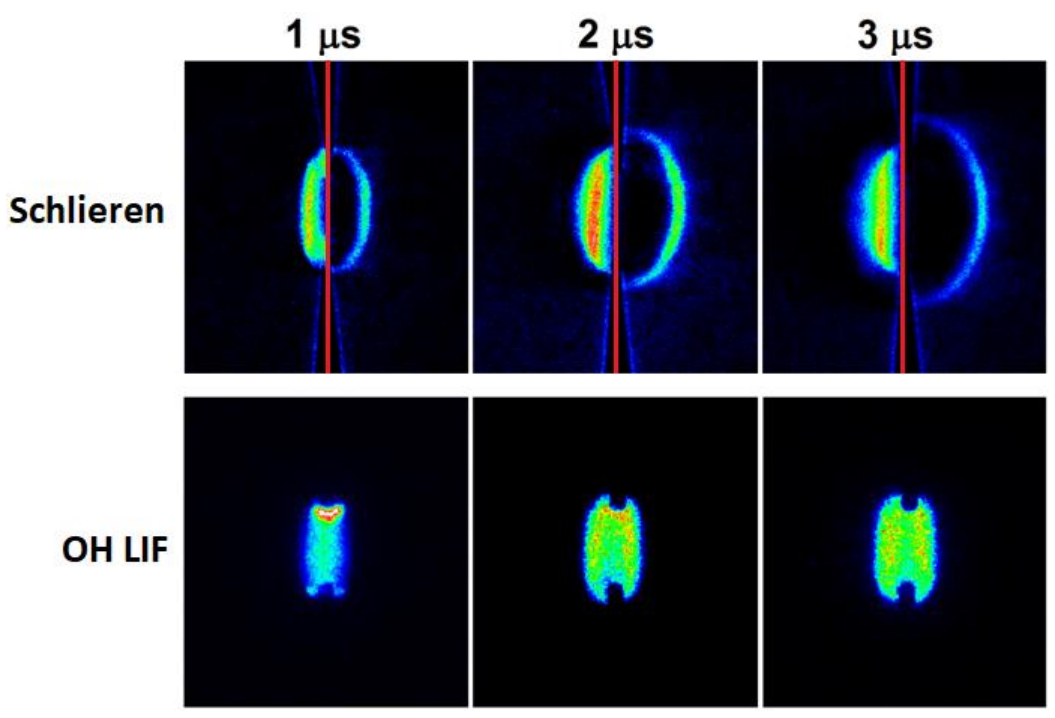

Figure 6. Schlieren and OH LIF images obtained in the afterglow of a single nanosecond spark. The discharge was initiated in ambient air by a single pulse of 10_-ns duration and 30--kV amplitude.
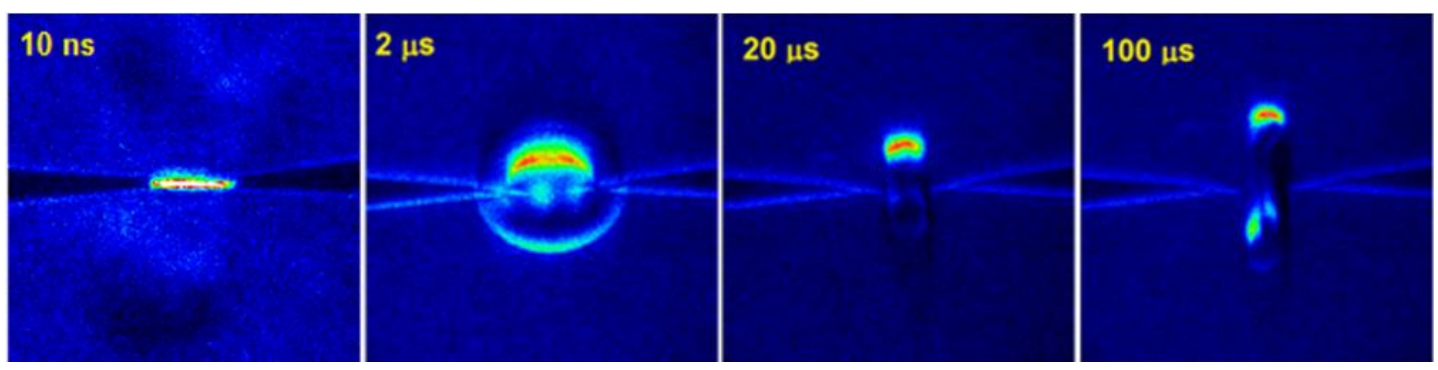

Figure 7. Schlieren images obtained in the long afterglow of a single nanosecond spark. The discharge was initiated in ambient air by a single pulse of $10_{-}-\mathrm{ns}$ duration and $30_{-}-\mathrm{kV}$ amplitude.

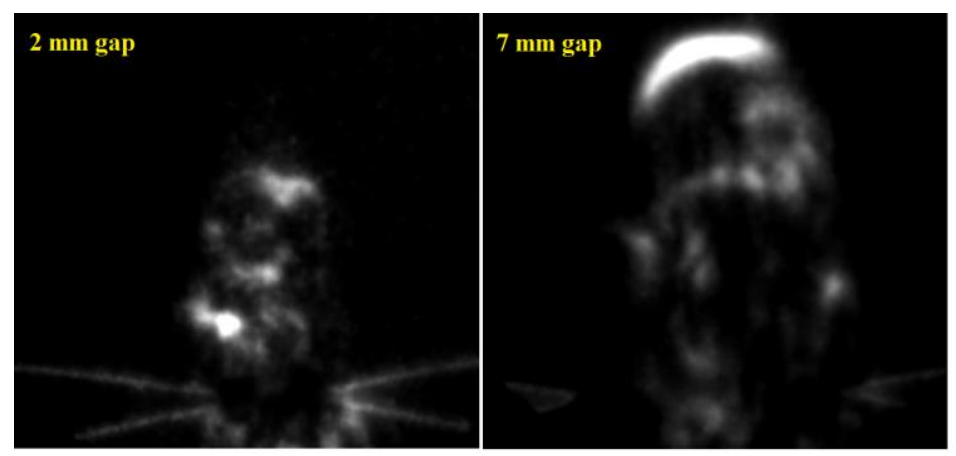

Figure 8. Schlieren images for a burst of 30 sparks at $30 \mathrm{kHz}$ for a $22--\mathrm{mm}$ inter-electrode gapbetween the electrodes (to the left) and a 7 --mm gap (to the right). Each pulse was of 10--ns duration and 30--kV amplitude. The discharge was initiated in ambient air. Both images were obtained at $1000 \mu \mathrm{s}$. 